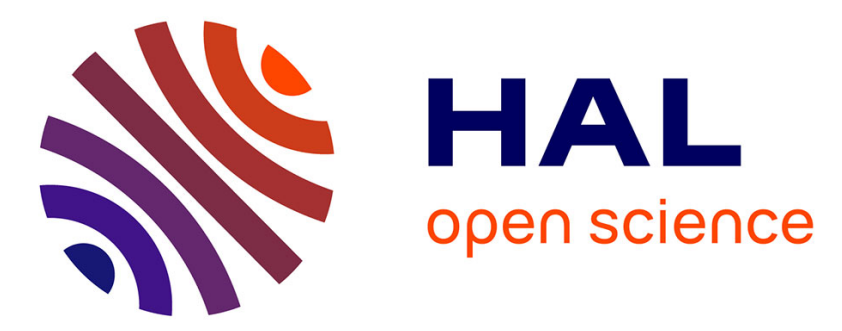

\title{
Automatic Image Annotation Based on Semi-supervised Probabilistic CCA
}

Bo Zhang, Gang Ma, Xi Yang, Zhongzhi Shi, Jie Hao

\section{To cite this version:}

Bo Zhang, Gang Ma, Xi Yang, Zhongzhi Shi, Jie Hao. Automatic Image Annotation Based on Semi-supervised Probabilistic CCA. 9th International Conference on Intelligent Information Processing (IIP), Nov 2016, Melbourne, VIC, Australia. pp.211-221, 10.1007/978-3-319-48390-0_22 . hal01614988

\section{HAL Id: hal-01614988 \\ https://hal.inria.fr/hal-01614988}

Submitted on 11 Oct 2017

HAL is a multi-disciplinary open access archive for the deposit and dissemination of scientific research documents, whether they are published or not. The documents may come from teaching and research institutions in France or abroad, or from public or private research centers.
L'archive ouverte pluridisciplinaire HAL, est destinée au dépôt et à la diffusion de documents scientifiques de niveau recherche, publiés ou non, émanant des établissements d'enseignement et de recherche français ou étrangers, des laboratoires publics ou privés. 


\title{
Automatic Image Annotation Based on Semi-supervised Probabilistic CCA ${ }^{\star}$
}

\author{
Bo Zhang ${ }^{1,2}$, Gang $\mathbf{M a}^{2,3}$, Xi Yang ${ }^{2}$, Zhongzhi Shi ${ }^{2}$, Jie Hao ${ }^{4}$ \\ ${ }^{1}$ China University of Mining and Technology, Xuzhou 221116, China \\ ${ }^{2}$ Institute of Computing Technology, Chinese Academy of Sciences, Beijing 100190, \\ China \\ ${ }^{3}$ University of Chinese Academy of Sciences, Beijing 100190, China \\ ${ }^{4}$ Xuzhou Medical University, School of Medicine Information, Xuzhou 221000, China \\ \{zhangb, mag, yangx, shizz\}@ics.ict.ac.cn \\ haojie@xzmc.edu.cn
}

\begin{abstract}
We propose a novel semi-supervised method for building a statistical model that represents the relationship between images and text labels (tags) based on a semi-supervised variant of CCA called SemiPC$\mathrm{CA}$, which extends the probabilistic CCA model to make use of the labelled and unlabelled images together to extract the low-dimensional latent space representing topics of images. Real-world image tagging experiments indicate that our proposed method improves the accuracy even when only a small number of labelled images are available.
\end{abstract}

Keywords: probabilistic CCA, semi-supervised method, automatic image annotation

\section{Introduction}

Automatic image annotation has become an important and challenging problem due to the existence of semantic gap. The state-of-the-art techniques of image auto-annotation can be roughly categorized into two different schools of thought. The first one defines auto-annotation as a traditional supervised classification problem, which treats each word (or semantic concept) as an independent class and creates different classifiers for every word. This approach computes similarity at the visual level and annotates a new image by propagating the corresponding words. The second perspective takes a different stand and treats images and texts as equivalent data. It attempts to discover the correlation between visual features and textual words on an unsupervised basis, by estimating the joint distribution of features and words. Thus, it poses annotation as statistical inference in a

\footnotetext{
* This work is supported by the National Program on Key Basic Research Project(973 Program)(No. 2013CB329502), National Natural Science Foundation of China(No.61035003), National High-tech R\&D Program of China(863 Program)(No.2012AA011003), National Science and Technology Support Program(No.2012BA107B02), Natural Science Foundation of Jiangsu Province(No.BK20160276).
} 
graphical model. Under this perspective, images are treated as bags of words and features, each of which are assumed generated by a hidden variable. Various approaches differ in the definition of the states of the hidden variable: some associate them with images in the database, while others associate them with image clusters or latent aspects (topics) .

As latent aspect models, PLSA [8] and latent Dirichlet allocation (LDA) [3] have been successfully applied to annotate and retrieve images. PLSA-WORDS [12] is a representative approach, which achieves the annotation task by constraining the latent space to ensure its consistency in words. However, since standard PLSA can only handle discrete quantity (such as textual words), this approach quantizes feature vectors into discrete visual words for PLSA modeling. Therefore, its annotation performance is sensitive to the clustering granularity. GM-PLSA [11] deals with the data of different modalities in terms of their characteristics, which assumes that feature vectors in an image are governed by a Gaussian distribution under a given latent aspect other than a multinomial one, and employs continuous PLSA and standard PLSA to model visual features and textual words respectively. This model learns the correlation between these two modalities by an asymmetric learning approach and then it can predict semantic annotation precisely for unseen images.

Canonical correlation analysis (CCA) is a data analysis and dimensionality reduction method similar to PCA. While PCA deals with only one data space, CCA is a technique for joint dimensionality reduction across two spaces that provide heterogeneous representations of the same data. CCA is a classical but still powerful method for analyzing these paired multi-view data. Since CCA can be interpreted as an approximation to Gaussian PLSA and also be regarded as an extension of Fisher linear discriminant analysis (FDA) to multi-label classification [1], learning topic models through CCA is not only computationally efficient, but also promising for multi-label image annotation and retrieval.

However, CCA requires the data be rigorously paired or one-to-one correspondence among different views due to its correlation definition. However, such requirement is usually not satisfied in real-world applications due to various reasons. To cope with this problem, several extensions of CCA have been proposed to utilize the meaningful prior information hidden in additional unpaired data. Blaschko et al. [2] proposes semi-supervised Laplacian regularization of kernel canonical correlation (SemiLRKCCA) to find a set of highly correlated directions by exploiting the intrinsic manifold geometry structure of all data (paired and unpaired). SemiCCA [10] resembles the manifold regularization, i.e., using the global structure of the whole training data including both paired and unpaired samples to regularize CCA. Consequently, SemiCCA seamlessly bridges CCA and principal component analysis (PCA), and inherits some characteristics of both PCA and CCA. Gu et al. [6] proposed partially paired locality correlation analysis (PPLCA), which effectively deals with the semi-paired scenario of wireless sensor network localization by virtue of the combination of the neighbourhood structure information in data. Most recently, Chen et al. [4] presents a general dimensionality reduction framework for semi-paired and semi-supervised 
multi-view data which naturally generalizes existing related works by using different kinds of prior information. Based on the framework, they develop a novel dimensionality reduction method, termed as semi-paired and semi-supervised generalized correlation analysis (S2GCA), which exploits a small amount of paired data to perform CCA.

We propose a semi-supervised variant of CCA named SemiPCCA based on the probabilistic model for CCA. The estimation of SemiPCCA model parameters is affected by the unpaied multi-view data (e.g. unlabelled image) which revealed the global structure within each modality. Then, an automatic image annotation method based on SemiPCCA is presented. Through estimating the relevance between images and words by using the labelled and unlabelled images together, this method is shown to be more accurate than previous publish methods.

This paper is organized as follows. After introducing the framework of the proposed SemiPCCA model briefly in Section 2, we formally present our automatic image annotation method based on SemiPCCA in Section 3. Finally Section 4 illustrates experiments results and Section 5 concludes the paper.

\section{Framework}

In this section, we first review a probabilistic model for CCA. Then armed with this probabilistic reformulation of CCA, we present our semi-supervised variant of CCA named SemiPCCA based on the probabilistic model for CCA. The estimation of SemiPCCA model parameters is affected by the unlabelled multi-view data which revealed the global structure within each modality.

\subsection{Probabilistic Canonical Correlation Analysis}

In [1], Bach and Jordan propose a probabilistic interpretation of CCA. In this model, two random vectors $x_{1} \in \mathbb{R}^{m_{1}}$ and $x_{2} \in \mathbb{R}^{m_{2}}$ are considered generated by the same latent variable $z \in \mathbb{R}^{d}\left(\min \left(m_{1}, m_{2}\right) \geqslant d \geqslant 1\right)$ and thus the "correlated" to each other.

In this model, the observations of $x_{1}$ and $x_{2}$ are generated form the same latent variable $z$ (Gaussian distribution with zero mean and unit variance) with unknown linear transformations $W_{1}$ and $W_{2}$ by adding Gaussian noise $\varepsilon_{1}$ and $\varepsilon_{2}$, i.e.,

$$
\begin{gathered}
P(z) \sim \mathcal{N}\left(0, I_{d}\right), \\
P\left(\varepsilon_{1}\right) \sim \mathcal{N}\left(0, \Psi_{1}\right), P\left(\varepsilon_{2}\right) \sim \mathcal{N}\left(0, \Psi_{2}\right), \\
x_{1}=W_{1} z+\mu_{1}+\varepsilon_{1}, W_{1} \in \mathbb{R}^{m_{1} \times d}, \\
x_{2}=W_{2} z+\mu_{2}+\varepsilon_{2}, W_{2} \in \mathbb{R}^{m_{2} \times d} .
\end{gathered}
$$


From [1], the corresponding maximum-likelihood estimations to the unknown parameters $\mu_{1}, \mu_{2}, W_{1}, W_{2}, \Psi_{1}$ and $\Psi_{2}$ are

$$
\begin{gathered}
\hat{\mu}_{1}=\frac{1}{N} \sum_{i=1}^{N} x_{1}^{2}, \hat{\mu}_{2}=\frac{1}{N} \sum_{i=1}^{N} x_{2}^{2}, \\
\hat{W}_{1}=\widetilde{\Sigma}_{11} U_{1 d} M_{1}, \hat{W}_{2}=\widetilde{\Sigma}_{22} U_{2 d} M_{2}, \\
\hat{\Psi}_{1}=\widetilde{\Sigma}_{11}-\hat{W}_{1} \hat{W}_{1}^{T}, \hat{\Psi}_{2}=\widetilde{\Sigma}_{22}-\hat{W}_{2} \hat{W}_{2}^{T},
\end{gathered}
$$

where $\widetilde{\Sigma}_{11}, \widetilde{\Sigma}_{22}$ have the same meaning of standard CCA, the columns of $U_{1 d}$ and $U_{2 d}$ are the first $d$ canonical directions, $P_{d}$ is the diagonal matrix with its diagonal elements given by the first $d$ canonical correlations and $M_{1}, M_{2} \in \mathbb{R}^{d \times d}$, with spectral norms smaller the one, satisfying $M_{1} M_{2}^{T}=P_{d}$. In our expectations, let $M_{1}=M_{2}=\left(P_{d}\right)^{1 / 2}$. The posterior expectations of $z$ given $x_{1}$ and $x_{2}$ are

$$
\begin{aligned}
& E\left(z \mid x_{1}\right)=M_{1}^{T} U_{1 d}^{T}\left(x_{1}-\hat{\mu}_{1}\right), \\
& E\left(z \mid x_{2}\right)=M_{2}^{T} U_{2 d}^{T}\left(x_{2}-\hat{\mu}_{2}\right) .
\end{aligned}
$$

Thus, $E\left(z \mid x_{1}\right)$ and $E\left(z \mid x_{2}\right)$ lie in the $d$ dimensional subspace that are identical with those of standard CCA.

\subsection{Semi-supervised PCCA}

Consider a set of paired samples of size $N_{p}, X_{1}^{P}=\left\{\left(x_{1}^{i}\right)\right\}_{i=1}^{N^{p}}$ and $X_{2}^{P}=$ $\left\{\left(x_{2}^{i}\right)\right\}_{i=1}^{N^{p}}$, where each sample $x_{1}^{i}$ (resp. $\left.x_{2}^{i}\right)$ is represented as a vector with dimension of $m_{1}$ (resp. $m_{2}$ ). When the number of paired of samples is small, CCA tends to overfit the given paired samples. Here, let us consider the situation where unpaired samples $X_{1}^{U}=\left\{\left(x_{1}^{j}\right)\right\}_{j=N^{p}+1}^{N^{1}}$ and/or $X_{2}^{U}=\left\{\left(x_{2}^{k}\right)\right\}_{k=N^{p}+1}^{N^{2}}$ are additional provided, where $X_{1}^{U}$ and $X_{2}^{U}$ might be independently generated. Since the original CCA and PCCA cannot directly incorporate such unpaired samples, we proposed a novel method named Semi-supervised PCCA (SemiPCCA) that can avoid overfitting by utilizing the additional unpaired samples. See Figure 1 for an illustration of the graphical model of the SemiPCCA model.

The whole observation is now $D=\left\{\left(x_{1}^{i}, x_{2}^{i}\right)\right\}_{i=1}^{N^{p}} \cup\left\{\left(x_{1}^{j}\right)\right\}_{j=N^{p}+1}^{N^{1}} \cup\left\{\left(x_{2}^{k}\right)\right\}_{k=N^{p}+1}^{N^{2}}$. The likelihood, with the independent assumption of all the data points, is calculated as

$$
L(\Theta)=\prod_{i=1}^{N^{p}} P\left(x_{1}^{i}, x_{2}^{i} ; \Theta\right) \prod_{j=N^{p}+1}^{N^{1}} P\left(x_{1}^{j} ; \Theta\right) \prod_{k=N^{p}+1}^{N^{2}} P\left(x_{2}^{k} ; \Theta\right)
$$

In SemiPCCA model, for paired samples $\left\{\left(x_{1}^{i}, x_{2}^{i}\right)\right\}_{i=1}^{N^{p}}, x_{1}^{i}$ and $x_{2}^{i}$ are considered generated by the same latent variable $z^{i}$ and $P\left(x_{1}^{i}, x_{2}^{i}\right)$ is calculated as in PCCA model, i.e.

$$
P\left(x_{1}^{i}, x_{2}^{i} ; \Theta\right) \sim \mathcal{N}\left(\left(\begin{array}{c}
\mu_{1} \\
\mu_{2}
\end{array}\right),\left(\begin{array}{cc}
W_{1} W_{1}^{T}+\Psi_{1} & W_{1} W_{2}^{T} \\
W_{2} W_{1}^{T} & W_{2} W_{2}^{T}+\Psi_{2}
\end{array}\right)\right) .
$$




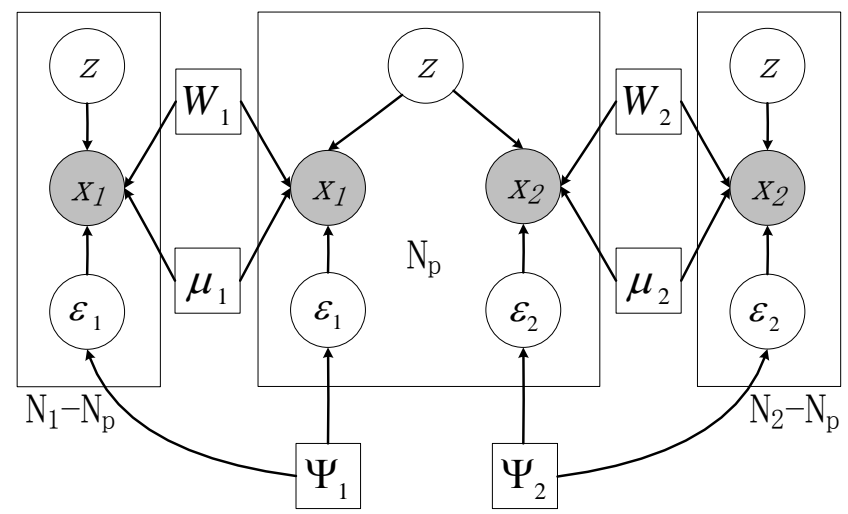

Fig. 1. Graphical model for Semi-supervised PCCA. The box denotes a plate comprising a data set of $N_{p}$ paired observations, and additional unpaired samples.

Whereas for unpaired observations $X_{1}^{U}=\left\{\left(x_{1}^{j}\right)\right\}_{j=N^{p}+1}^{N^{1}}$ and/or $X_{2}^{U}=\left\{\left(x_{2}^{k}\right)\right\}_{k=N^{p}+1}^{N^{2}}$, $x_{1}^{j}$ and $x_{2}^{k}$ are separately generated from the latent variable $z_{1}{ }^{j}$ and $z_{2}{ }^{k}$ with linear transformations $W_{1}$ and $W_{2}$ by adding Gaussian noise $\varepsilon_{1}$ and $\varepsilon_{2}$. From Eq.(1),

$$
\begin{aligned}
& P\left(x_{1}^{j} ; \Theta\right) \sim \mathcal{N}\left(\mu_{1}, W_{1} W_{1}^{T}+\Psi_{1}\right), \\
& P\left(x_{2}^{k} ; \Theta\right) \sim \mathcal{N}\left(\mu_{2}, W_{2} W_{2}^{T}+\Psi_{2}\right) .
\end{aligned}
$$

For means of $x_{1}$ and $x_{2}$ we have

$$
\hat{\mu_{1}}=\frac{1}{N_{1}} \sum_{i=1}^{N_{1}} x_{1}^{i}, \hat{\mu_{2}}=\frac{1}{N_{2}} \sum_{i=1}^{N_{2}} x_{1}^{i},
$$

which are just the sample means. Since they are always the same in all EM iterations, we can centre the data $X_{1}^{P} \cup X_{1}^{U}, X_{2}^{P} \cup X_{2}^{U}$ by subtracting these means in the beginning and ignore these parameters in the learning process. So for simplicity we change the notation $x_{1}^{i}, x_{2}^{i}, x_{1}^{j}$ and $x_{2}^{k}$ to be the centred vectors in the following.

For the two mapping matrices, we have the updates

$$
\begin{aligned}
& \hat{W}_{1}=\left(\sum_{i=1}^{N_{p}} x_{1}^{i}\left\langle z^{i}\right\rangle^{T}+\sum_{j=N_{p}+1}^{N_{1}} x_{1}^{j}\left\langle z_{1}{ }^{j}\right\rangle^{T}\right)\left(\sum_{i=1}^{N_{p}}\left\langle z^{i} z^{i^{T}}\right\rangle+\sum_{j=N_{p}+1}^{N_{1}}\left\langle z_{1}{ }^{j} z_{1}{ }^{T}\right\rangle\right)^{-1} \\
& \hat{W}_{2}=\left(\sum_{i=1}^{N_{p}} x_{2}^{i}\left\langle z^{i}\right\rangle^{T}+\sum_{k=N_{p}+2}^{N_{2}} x_{2}^{k}\left\langle z_{2}{ }^{k}\right\rangle^{T}\right)\left(\sum_{i=1}^{N_{p}}\left\langle z^{i} z^{i T}\right\rangle+\sum_{k=N_{p}+1}^{N_{2}}\left\langle z_{2}{ }^{k} z_{2}{ }^{k T}\right\rangle\right)
\end{aligned}
$$


Finally the noise levels are updated as

$$
\begin{gathered}
\hat{\Psi}_{1}=\frac{1}{N_{1}}\left\{\left(\sum_{i=1}^{N_{p}}\left(x_{1}^{i}-\hat{W}_{1}\left\langle z^{i}\right\rangle\right)\left(x_{1}^{i}-\hat{W}_{1}\left\langle z^{i}\right\rangle\right)^{T}+\right.\right. \\
\left.\left.\sum_{j=N_{p}+1}^{N_{1}}\left(x_{1}^{j}-\hat{W}_{1}\left\langle z_{1}{ }^{j}\right\rangle\right)\left(x_{1}^{j}-\hat{W}_{1}\left\langle z_{1}{ }^{j}\right\rangle\right)^{T}\right)\right\} \\
\hat{\Psi}_{2}=\frac{1}{N_{2}}\left\{\left(\sum_{i=1}^{N_{p}}\left(x_{2}^{i}-\hat{W}_{2}\left\langle z^{i}\right\rangle\right)\left(x_{2}^{i}-\hat{W}_{2}\left\langle z^{i}\right\rangle\right)^{T}\right.\right. \\
\left.\left.+\sum_{k=N_{p}+1}^{N_{2}}\left(x_{2}^{k}-\hat{W}_{2}\left\langle z_{2}{ }^{k}\right\rangle\right)\left(x_{2}^{k}-\hat{W}_{2}\left\langle z_{2}{ }^{k}\right\rangle\right)^{T}\right)\right\}
\end{gathered}
$$

\subsection{Projections in SemiPCCA Model}

Analogous to the PCCA model, the projection of a labelled image $\left(x_{1}^{i}, x_{2}^{i}\right)$ in SemiPCCA model is directly given by Eq.(3).

Although this result looks similar as that in PCCA model, the learning of $W_{1}$ and $W_{2}$ are influenced by those unpaired samples. Unpaired samples reveal the global structure of whole the samples in each domain. Note once a basis in one sample space is rectified, the corresponding bases in the other sample space is also rectified so that correlations between two bases are maximized.

\section{Annotation on unlabelled image}

Now, we presents an automatic image annotation method based on the SemiPC$\mathrm{CA}$, which estimating the association between images and words by using the labelled and unlabelled images together.

Let $X_{1}^{P}=\left\{\left(x_{1}^{i}\right)\right\}_{i=1}^{N^{p}}$ and $X_{2}^{P}=\left\{\left(x_{2}^{i}\right)\right\}_{i=1}^{N^{p}}$ be the set of labelled images and its corresponding semantic features with $m_{1}$ and $m_{2}$ dimensions of size $N_{p}$, and $X_{1}^{U}=\left\{\left(x_{1}^{j}\right)\right\}_{j=N^{p}+1}^{N^{1}}$ be a set of unlabelled images.

The first step is to extracts image features and labels features of training samples, and generates the essential latent space by fitting SemiPCCA.

In the context of automatic image annotation, $X_{1}^{U}$ only exists, whereas $X_{2}^{U}$ is empty. So, for the mapping matrices $W_{2}$ and the noise levels $\Psi_{2}$, we have to change the updates as follows,

$$
\begin{gathered}
\hat{W}_{2}=\left(\sum_{i=1}^{N_{p}} x_{2}^{i}\left\langle z^{i}\right\rangle^{T}\right)\left(\sum_{i=1}^{N_{p}}\left\langle z^{i} z^{i^{T}}\right\rangle\right)^{-1} \\
\hat{\Psi}_{2}=\frac{1}{N_{p}}\left\{\left(\sum_{i=1}^{N_{p}}\left(x_{2}^{i}-\hat{W}_{2}\left\langle z^{i}\right\rangle\right)\left(x_{2}^{i}-\hat{W}_{2}\left\langle z^{i}\right\rangle\right)^{T}\right)\right\}
\end{gathered}
$$


Using this model, we derive the posterior probability of a sample in the latent space. When only an image feature $x_{1}$ is given, the posterior probability $P\left(z_{1} \mid x_{1}\right)$ of estimated latent variable $z_{1}$ becomes a normal distribution whose mean and variance are,

$$
\begin{aligned}
& \mu_{z_{1}}=\hat{W}_{1}^{T}\left(\hat{W}_{1} \hat{W}_{1}^{T}+\hat{\Psi}_{1}\right)^{-1}\left(x_{1}-\hat{\mu}_{1}\right), \\
& \Psi_{z_{1}}=I-\hat{W}_{1}^{T}\left(\hat{W}_{1} \hat{W}_{1}^{T}+\hat{\Psi}_{1}\right)^{-1}
\end{aligned}
$$

respectively. Also, when both an image feature $x_{1}$ and semantic feature $x_{2}$ are given, the posterior probability $P\left(z \mid x_{1}, x_{2}\right)$ becomes,

$$
\begin{aligned}
& \mu_{z}=\hat{W}^{T}\left(\hat{W} \hat{W}^{T}+\hat{\Psi}\right)^{-1}\left(\left(\begin{array}{l}
x_{1} \\
x_{2}
\end{array}\right)-\hat{\mu}\right), \\
& \Psi_{z}=I-\hat{W}^{T}\left(\hat{W} \hat{W}^{T}+\hat{\Psi}\right)^{-1} \hat{W}
\end{aligned}
$$

The second step is to map labelled training images $\left\{T_{i}^{(P)}=\left(x_{1}^{i}, x_{2}^{i}\right)\right\}_{i=1}^{N^{p}}$ and unlabelled images $\left\{\left(Q_{j}^{(U)}=x_{1}^{j}\right\}_{j=N^{p}+1}^{N^{1}}\right.$ to the latent space with posterior probability $P\left(z \mid x_{1}, x_{2}\right)$ and $P\left(z \mid x_{1}\right)$ separately, and $\mathrm{K}-\mathrm{L}$ distance is used for measuring the similarity between two images.

We define the similarity between two samples as follows. When two labelled images $T_{i}^{(P)}=\left(x_{1}^{i}, x_{2}^{i}\right)$ and $T_{j}^{(P)}=\left(x_{1}^{j}, x_{2}^{j}\right)$ are available, then similarity is defined as,

$$
D\left(T_{i}^{(P)}, T_{j}^{(P)}\right)=\left(\mu_{z}^{i}-\mu_{z}^{j}\right)^{T} \Psi_{z}^{-1}\left(\mu_{z}^{i}-\mu_{z}^{j}\right)
$$

which measuring essential similarities both in terms of appearance and semantics.

Furthermore, when labels feature of one of two samples is not available, e.g. one labelled image $T_{i}^{(P)}=\left(x_{1}^{i}, x_{2}^{i}\right)$ and one unlabelled image $Q_{j}^{(U)}=x_{1}^{j}$, which is the usual case in automatic image annotation, our framework also enables measuring similarities with semantic aspects even in the absence of labels features, and their similarity becomes:

$$
D\left(T_{i}^{(P)}, Q_{j}^{(U)}\right)=\left(\mu_{z}^{i}-\mu_{z_{1}}^{j}\right)^{T}\left(\frac{\Psi_{z}^{-1}+\Psi_{z_{1}}^{-1}}{2}\right)\left(\mu_{z}^{i}-\mu_{z_{1}}^{j}\right)
$$

As we described, we can formalize a new image annotation method. Let $x_{n e w}$ demote a newly input image. To annotate $x_{n e w}$ with some words, we calculate the posterior probability posterior probability of a word $w$ given by $x_{n e w}$, which is represented as

$$
P\left(w \mid x_{n e w}\right)=\sum_{i=1}^{N_{p}} P\left(w \mid T_{i}^{(P)}\right) P\left(T_{i}^{(P)} \mid x_{n e w}\right) .
$$


The posterior probability $P\left(T_{i}^{(P)} \mid x_{\text {new }}\right)$ of each labelled image $T_{i}^{(P)}$ using the above similarity measurement is defined as follow,

$$
P\left(T_{i}^{(P)} \mid x_{\text {new }}\right)=\frac{\exp \left(-D\left(T_{i}^{(P)}, x_{\text {new }}\right)\right)}{\sum_{i=1}^{N_{p}} \exp \left(-D\left(T_{i}^{(P)}, x_{\text {new }}\right)\right)},
$$

where, the denominator is a regularization term so that $\sum_{i=1}^{N_{p}} P\left(T_{i}^{(P)} \mid x_{\text {new }}\right)=$ 1. $P\left(w \mid T_{i}^{(P)}\right)$ corresponds to the sample-to-label model, which is defined as

$$
P\left(w \mid T_{i}^{(P)}\right)=\mu \delta_{w, T_{i}^{(P)}}+(1-\mu) \frac{N_{w}}{N W},
$$

where $N_{w}$ is the number of the images that contain $w$ in the training data set, $\delta_{w, T_{i}^{(P)}}=1$ if word $w$ is annotated in the training sample $T_{i}^{(P)}$, otherwise $\delta_{w, T_{i}^{(P)}} \stackrel{2}{=} 0 . \mu$ is a parameter between zero and one. $N W$ is the number of the words.

The words are sorted in descending order of the posterior probability $P\left(w \mid x_{n e w}\right)$. The highest ranked words are used to annotate the image $x_{n e w}$.

\section{Experiments}

This section describes the results for the automatic image annotation task.

We use Corel5K and Corel30K to evaluate the performance of the proposed method. Corel5K contains 5,000 pairs of the image and the labels. Each image is manually annotated with one to five words. The training data has 371 words. 260 words among them appear in the test data.

Corel30K dataset is an extension of the Corel5K dataset based on a substantially larger database, which tries to correct some of the limitation in Corel5k such as small number of examples and small size of the vocabulary. Corel30K dataset contains 31,695 images and 5,587 words.

We follow the methodology of previous works, 500 images from the Corel5K are the test data. The other 1500, 2250 and 4500 images are selected from the Corel5K as the training data respectively, alone with the remaining training images in Corel5K and 31,695 images in Corel30K which acted as the unlabelled image to estimate the parameters of SemiPCCA together.

\subsection{Feature Representation}

As the image feature, we use the color higher-order local auto-correlation (ColorHLAC) features. This is a powerful global image feature for color images. Generally, global image features are suitable for realizing scalable systems because they can be extracted quite fast. Also, they are well suited for unconstrained image level annotation. 
The Color-HLAC features enumerate all combinations of mask patterns that define autocorrelations of neighboring points and include both color information and texture information simultaneously. In this paper we use at most the $2 \mathrm{nd}$ order correlations, whose dimension is 714 . The 2nd order Color-HLAC feature is reduced by PCA to preserve the 80 dimensions.

We extract Color-HLAC features from two scales $(1 / 1,1 / 2$ size) to obtain robustness against scale change. Also, we extract them from edge images obtained by using the Sobel filter as well as the normal images. In all, the final image features are 320 dimensions.

As for labels feature, we use the word histogram. In this work, each image is simply annotated with a few words, so the word histogram becomes a binary feature.

\subsection{Evaluation and results}

In this section, the performance of our model (SemiPCCA) is compared with several models. Image annotation performance is evaluated by comparing the captions automatically generated for the test set with the human-produced ground truth. For evaluation of annotation performance of our method, we follow the methodology of previous works. We define the automatic annotation as the five semantic words of largest posterior probability, and compute the recall and precision of every word in the test set. For a given semantic word, recall $=\mathrm{B} / \mathrm{C}$ and precision $=\mathrm{B} / \mathrm{A}$, where $\mathrm{A}$ is the number of images automatically annotated with a given word; $\mathrm{B}$ is the number of images correctly annotated with that word; $\mathrm{C}$ is the number of images having that word in ground truth annotation. The average word precision and word recall values summarize the system performance.

Table 1 shows the results obtained by the proposed method and various previously proposed methods - - CRM [9],MBRM [5], PLSA-WORDS [12], GMPLSA [11], using Corel5K. In order to compare with those previous models, we divide this dataset into 2 parts: a training set of 4,500 images and a test set of 500 images. We report the results on two sets of words: the subset of 49 best words and the complete set of all 260 words that occur in the training set. From the table, we can see that our model performs significantly better than all other models. We believe that using SemiPCCA to model visual and textual data by labelled and unlabelled images respectively is the reason for this result.

\section{Conclusions}

This paper presents an automatic image annotation method based on the SemiPCCA. Through estimating the association between images and words by using the labelled and unlabelled images together, this method is shown to be more accurate than previous publish methods. Experiments on the Corel dataset prove that our approach is promising for semantic image annotation. In comparison to several state-of-the-art annotation models, higher accuracy and superior effectiveness of our approach are reported. 
Table 1. Performance comparison of different automatic image annotation models on Corel5k dataset.

\begin{tabular}{|c|c|c|c|c|c|}
\hline Models & CRM & MBRM & PLSA WORDS & GM PLSA & Semi PCCA \\
\hline \#Words with recall $>0$ & 107 & 122 & 105 & 125 & 151 \\
\hline \multicolumn{7}{|c|}{ Results on 49 best words, } \\
\hline MR & 0.70 & 0.78 & 0.71 & 0.79 & 0.94 \\
\hline MP & 0.59 & 0.74 & 0.56 & 0.76 & 0.77 \\
\hline F1 & 0.64 & 0.76 & 0.63 & 0.77 & 0.85 \\
\hline MR & 0.19 & 0.25 & 0.20 & 0.25 & 0.32 \\
\hline MP & 0.24 & 0.24 & 0.14 & 0.26 & 0.24 \\
\hline F1 & 0.21 & 0.24 & 0.16 & 0.25 & 0.27 \\
\hline
\end{tabular}

\section{References}

1. Francis R. Bach and Michael I. Jordan. A probability interpretation of canonical correlation analysis. Technical Report Technical Report 688, Department of Statistics, Universityof California, Berkeley, 2005.

2. Matthew Blaschko, Christoph Lampert, and Arthur Gretton. Semi-supervised laplacian regularization of kernel canonical correlation analysis. In ECML PKDD 2008, pages 133-145, Antwerp, Belgium, 2008. Springer Verlag.

3. David M. Blei, Andrew Y. Ng, and Michael I. Jordan. Latent dirichlet allocation. Journal of Machine Learning Research, 3(4-5):993-1022, 2003.

4. Xiaohong Chen, Songcan Chen, Hui Xue, and Xudong Zhou. A unified dimensionality reduction framework for semi-paired and semi-supervised multiview data. Pattern Recognition, 45(5):2005-2018, 2012.

5. S. L. Feng, R. Manmatha, and V. Lavrenko. Multiple bernoulli relevance models for image and video annotation. In CVPR04, pages 1002-1009, Washington, DC, United states, 2004.

6. Jingjing Gu, Songcan Chen, and Tingkai Sun. Localization with incompletely paired data in complex wireless sensor network. IEEE Transactions on Wireless Communications, 10(9):2841-2849, 2011.

7. Tatsuya Harada, Hideki Nakayama, and Yasuo Kuniyoshi. Image annotation and retrieval based on efficient learning of contextual latent space. In ICME09, pages 858-861, Piscataway, USA, 2009.

8. Thomas Hofmann. Unsupervised learning by probabilistic latent semantic analysis. Machine Learning, 42:177-196, 2001.

9. J. Jeon, V. Lavrenko, and R. Manmatha. Automatic image annotation and retrieval using crossmedia relevance models. In SIGIR03, pages 119-126, Toronto, Canada, 2003.

10. Akisato Kimura, Hirokazu Kameoka, Masashi Sugiyama, and Takuho Nakano. Semicca: Efficient semi-supervised learning of canonical correlations. In ICPR10, pages 2933-2936, Istanbul, Turkey, 2010.

11. Zhixin Li, Zhiping Shi, Xi Liu, and Zhongzhi Shi. Modeling continuous visual features for semantic image annotation and retrieval. Pattern Recognition Letters, 32(3):516-523, 2011.

12. Florent Monay and Daniel Gatica-Perez. Modeling semantic aspects for crossmedia image indexing. IEEE Transactions on Pattern Analysis and Machine Intelligence, 29(10):1802-1817, 2007. 